\title{
Children hospitalized early and increased risk for future serious injury
}

\author{
H McPhillips, M Gallaher, T Koepsell
}

\begin{abstract}
Objective-To determine if infants hospitalized for any reason before 90 days of age are at increased risk for future serious injury.

Setting-Washington State.

Methods-A population based retrospective cohort study, using data from Washington State birth and death certificates linked to a statewide hospital discharge database for the years 1989 through 1997. Participants included healthy full term infants born in Washington State between 1989 and 1995. A total of 29466 infants hospitalized $<90$ days of age (early hospitalization) were compared to 29750 randomly selected infants not hospitalized early. The primary outcome was an injury resulting in hospitalization or death between 3-24 months.
\end{abstract}

Results-Among infants hospitalized early, 76/10 000 had a subsequent serious injury before age 2 , compared with 47 I 10000 infants without an early hospitalization (relative risk (RR) 1.6; 95\% confidence interval (CI) 1.3 to 2.0 ). In a multivariate model including maternal age and parity, the adjusted RR for serious injury associated with early hospitalization was $1.5(95 \%$ CI 1.2 to 1.8$)$. Infants hospitalized early were three times as likely to be hospitalized between 3-24 months of age for intentional injury compared with infants not hospitalized early (RR 3.3; 95\% CI 1.1 to 10.1 ).

Conclusions-Infants hospitalized in the first three months of life for any reason were $50 \%$ more likely to have a subsequent serious injury compared with infants not hospitalized early and were also at increased risk of intentional injury. This identifiable group of infants might be suitable for targeted childhood injury prevention programs including those involving prenatal and postnatal visits.

(Injury Prevention 2001;7:150-154)

Keywords: wounds and injuries; child abuse; hospitalization

Risk factors for dying from an injury in early childhood include low birth weight, young maternal age, less than 12 years of maternal education, higher parity, and low household income. ${ }^{12}$ The same sociodemographic characteristics are found in children at high risk for abuse. ${ }^{34}$ Interventions have been proven effective in reducing medical visits and hospital days due to injury in children living in very high risk families. ${ }^{5}{ }^{6}$ However, over $20 \%$ of infants in the
United States have two or more sociodemographic risk factors for dying from an injury. ${ }^{7}$ Targeting resource intensive injury prevention interventions to such a large high risk group is not feasible in many communities. If health care providers were better able to predict which children are at highest risk for injury, resources to prevent these injuries could be used more effectively.

Most serious injuries in early childhood fall somewhere on a continuum between those resulting from inadequate parental supervision or inadequate use of safety devices to neglect or outright abuse. Evidence exists that abused children are hospitalized more frequently for medical illness apparently unrelated to abuse than non-abused children, in some cases even before any abuse had been reported or suspected..$^{8-10}$

This study was designed to determine if infants hospitalized in the first three months of life are at increased risk of serious injury in early childhood, and would therefore be an important population to target for prevention measures.

\section{Methods}

We conducted a population based retrospective cohort study of healthy full term infants born in Washington State between 1989 and 1995. The study cohort was identified by linking birth certificates to the Comprehensive Hospital Abstract Reporting System (CHARS) database for the years 1989 to 1997. CHARS was created by the Washington State Department of Health and contains hospital discharge information for all hospitalizations in the state except those that occur in federal hospitals. Information available in the linked hospitalization record included date of admission and discharge, nine discharge diagnoses (International Classification of Diseases, Ninth Revision, Clinical Modification (ICD-9-CM) N codes), and one cause of injury code (ICD9-CM E code). Information on deaths due to injury, which included date and cause of death (ICD9-CM E code), was obtained by linking the birth certificates to death certificates for the years 1989 to 1997 .

Infants were included in the study if they were $\geqslant 37$ weeks gestational age, had a birth weight $\geqslant 2000 \mathrm{~g}$, and had a birth hospitalization lasting $\leqslant 7$ days. Infants were excluded from the study if they died before 90 days of age. Approximately 590000 infants were born in Washington State between 1989 and 1995. Our study sample included all 29466 healthy full term infants who met our inclusion criteria and who had a hospitalization for any reason before 90 days of age. We then randomly 
selected 29750 healthy full term infants from the birth cohort (frequency matched by birth year) who also met our inclusion criteria but who did not have an early hospitalization. We excluded premature infants because they are known to be at high risk for injury, ${ }^{7}$ are likely to be in the hospital for much of their first 90 days of life, and are also more likely to have professional follow up after hospital discharge.

MATERNAL/CHILD CHARACTERISTICS

We obtained other information regarding infant and maternal characteristics from the birth certificate and birth hospitalization discharge record that might confound or modify the association between early hospitalization and injury risk. Each characteristic was examined for confounding or effect modification by stratified analysis. Infant characteristics examined included the presence of birth defects, gender, and birth weight. Maternal characteristics examined were age at time of delivery, race, county of residence, marital status, parity before delivery of study infant, trimester prenatal care began, and smoking during pregnancy. Paternal characteristics examined were age and whether or not a father was listed on the birth certificate.

As maternal education was only available on birth certificates after 1991, we limited the analysis to infants born 1992-95 when examining maternal education as a potential confounder or effect modifier. The Washington State birth certificate also has employment information about the mother and father at the time of delivery. This information was combined into three mutually exclusive categories: employed, teen, or unemployed. Maternal and paternal employment were examined for confounding or effect modification by stratified analysis, although $9.2 \%$ of the data for maternal occupation and $22 \%$ of the data for paternal occupation were missing.

REASON FOR FIRST ADMISSION

We examined the first diagnosis on the hospital discharge record of the first hospitalization at less than 90 days of age to determine if the diagnosis was related to the risk of subsequent injury hospitalizations or death due to injury. Due to limitations in sample size, we grouped the early diagnoses into the following broad categories based on ICD-9-CM codes: nonrespiratory infection, pneumonia/bronchiolitis, jaundice, and perinatal conditions. All other reasons for the first admission were grouped as "other" such as diseases due to non-infectious gastrointestinal diseases, nutritional deficiencies, anemia, or malignancy.

OUTCOMES

The primary study outcome was death or hospitalization due to injury between 3 and 24 months of age. The first four hospitalizations occurring between 3 and 24 months of age were assessed, accounting for $98 \%$ of hospitalizations in both groups. In children with more than one hospitalization between 3 and 24 months, only the first injury hospitalization was counted.
As a secondary analysis, the risk of hospitalization or death by whether the injury was intentional or not was also assessed.

\section{ANALYSIS}

In the univariate and stratified analysis, we used the Mantel-Haenszel relative risk estimate and test based methods to obtain the $95 \%$ confidence interval (CI) for the risk of hospitalization or death due to injury among those infants hospitalized before 90 days compared with those who were not hospitalized during this time. On stratified analysis, significant confounding was defined by a change in the relative risk (RR) of $\geqslant 10 \%$ and effect modification was defined by a Breslow-Day test of homogeneity $\mathrm{p}$ value $<0.05$. Binomial regression with the log link function was conducted using the binreg command under Stata 6.0. This form of analysis yielded adjusted relative risks, which could be compared to unadjusted relative risks calculated from the bivariate analyses. All injury hospitalizations (intentional, unintentional, and those of undetermined intent) were included as a single dichotomous outcome variable in the final binomial regression model.

\section{Results}

Of infants who were hospitalized early, 225 (76/10 000) had a subsequent injury hospitalization or death due to injury, compared with 141 (47/10 000) of those who were not hospitalized early (RR 1.6; 95\% CI 1.3 to 2.0 ). Burns, ingestions, foreign bodies, and falls accounted for over $70 \%$ of all injury hospitalizations in both groups (table 1).

Over the seven year study period, six children in the early hospitalization group and eight in the control group died from injury related causes. The children who had an early hospitalization subsequently died from an ingestion, a drowning, suffocation, electrocution, and abuse (2). Those not hospitalized early died from a motor vehicle accident, a fall, an animal attack, drowning (3), and suffocation (2).

Infants who were hospitalized before 90 days of age (early hospitalization) differed from those who did not have an early hospitalization in many ways (table 2). Infants hospitalized early were more likely to be male, have a birth weight between 2000 and 2499 g, and were more likely to have a malformation. Mothers of infants hospitalized early were younger, more likely to be unmarried, unemployed, have less than a high school education, be on Medicaid, begin prenatal care late, have smoked during pregnancy, and were less likely to live in urban areas and to be white, non-Hispanic.

There was no evidence that any of the infant, maternal, or paternal characteristics that were examined importantly confounded or modified the relationship between early hospitalization and subsequent injury hospitalization or death. In multiple binomial regression, maternal age and parity were also found to be significant predictors of subsequent serious injury and were included in the final regression model 
Table 1 Selected newborn and maternal characteristics of healthy full term infants with a hospitalization in the first 90 days and comparison infants, Washington State 1989-1996

\begin{tabular}{|c|c|c|c|c|c|}
\hline & \multicolumn{2}{|c|}{$\begin{array}{l}\text { Early hospitalization } \\
(n=29466)\end{array}$} & \multicolumn{2}{|c|}{$\begin{array}{l}\text { No early hospitalization } \\
(n=29750)\end{array}$} & \multirow[b]{2}{*}{$\begin{array}{l}\text { Stratum specific } \\
R R(95 \% C I)\end{array}$} \\
\hline & No & $\begin{array}{l}\text { Injury } \\
\text { rate }\end{array}$ & No & $\begin{array}{l}\text { Injury } \\
\text { rate }\end{array}$ & \\
\hline All children & 29466 & 76 & 29750 & 47 & $1.6(1.3$ to 2.0$)$ \\
\hline Infant gender & & & & & $1.6(1.3$ to 2.0$) \dagger$ \\
\hline Male & 16821 & 84 & 15098 & 52 & $1.6(1.2$ to 2.1$)$ \\
\hline Female & 12645 & 70 & 14652 & 43 & $1.6(1.2$ to 2.3$)$ \\
\hline Birth weight (g) & & & & & $1.6(1.3$ to 2.0$) \dagger$ \\
\hline $2000-2499$ & 996 & 80 & 559 & 54 & $1.5(0.4$ to 5.6$)$ \\
\hline $2500-4000$ & 24708 & 78 & 24768 & 50 & $1.5(1.2$ to 1.9$)$ \\
\hline$>4000$ & 3752 & 77 & 4414 & 32 & $2.4(1.3$ to 4.6$)$ \\
\hline Any malformation at birth & 2202 & 77 & 1383 & 72 & $1.1(0.5$ to 2.3$)$ \\
\hline \multicolumn{6}{|l|}{ Maternal characteristics } \\
\hline Age (years) & & & & & $1.5(1.2$ to 1.9$) \dagger$ \\
\hline$<20$ & 4192 & 112 & 3039 & 79 & $1.4(0.9$ to 2.3$)$ \\
\hline $20-24$ & 8411 & 101 & 7492 & 63 & $1.6(1.1$ to 2.3$)$ \\
\hline $25-34$ & 14212 & 60 & 16113 & 39 & $1.5(1.1$ to 2.1$)$ \\
\hline $35+$ & 2633 & 30 & 3084 & 23 & $1.3(0.5$ to 3.7$)$ \\
\hline Race & & & & & $1.6(1.3$ to 2.0$) \dagger$ \\
\hline White, non-Hispanic & 22916 & 76 & 23683 & 46 & $1.6(1.3$ to 2.1$)$ \\
\hline White, Hispanic & 2678 & 49 & 2150 & 42 & $1.2(0.5$ to 2.7$)$ \\
\hline African American & 902 & 122 & 1027 & 58 & $2.1(0.8$ to 5.6$)$ \\
\hline Marital status & & & & & $1.6(1.3$ to 2.0$) \dagger$ \\
\hline Not married & 8659 & 98 & 6890 & 58 & $1.7(1.2$ to 2.5$)$ \\
\hline Married & 20763 & 70 & 22821 & 45 & $1.6(1.2$ to 2.0$)$ \\
\hline Education $\ddagger$ & & & & & $1.5(1.0$ to 2.1$) \dagger$ \\
\hline Less than high school & 1768 & 79 & 1295 & 61 & $1.3(0.7$ to 2.5$)$ \\
\hline High school & 3683 & 69 & 3824 & 56 & $1.2(0.7$ to 2.1$)$ \\
\hline$>$ High school & 4569 & 45 & 5689 & 20 & $2.3(1.1$ to 4.6$)$ \\
\hline Employment & & & & & $1.7(1.4$ to 2.1$) \dagger$ \\
\hline Teen & 1320 & 129 & 937 & 75 & $2.3(1.1$ to 4.6$)$ \\
\hline Unemployed & 11751 & 90 & 11313 & 55 & $1.6(1.2$ to 2.2$)$ \\
\hline Employed & 13496 & 62 & 14944 & 36 & $1.7(1.2$ to 2.4$)$ \\
\hline Source of insurance & & & & & $1.5(1.2$ to 1.9$) \dagger$ \\
\hline Medicaid & 11268 & 93 & 8293 & 58 & $1.6(1.1$ to 2.3$)$ \\
\hline Other & 16406 & 69 & 18336 & 46 & $1.5(1.1$ to 2.0$)$ \\
\hline County of residence & & & & & $1.6(1.3$ to 2.0$) \dagger$ \\
\hline Rural & 3799 & 97 & 3543 & 54 & $1.8(1.0$ to 3.2$)$ \\
\hline Suburban/small urban & 8451 & 64 & 7128 & 52 & $1.2(0.8$ to 1.9$)$ \\
\hline Metropolitan & 16948 & 78 & 18748 & 45 & $1.7(1.3$ to 2.3$)$ \\
\hline No of prior deliveries & & & & & $1.6(1.3$ to 2.0$) \dagger$ \\
\hline $2+$ & 7996 & 96 & 7694 & 55 & $1.8(1.2$ to 2.6$)$ \\
\hline 1 & 9522 & 83 & 9755 & 48 & $1.7(1.2$ to 2.5$)$ \\
\hline 0 & 11579 & 60 & 11975 & 42 & $1.4(1.0$ to 2.1$)$ \\
\hline Month prenatal care began & & & & & $1.6(1.3$ to 2.0$) \dagger$ \\
\hline $7-10$ & 1095 & 146 & 913 & 33 & $4.4(1.3$ to 15.2$)$ \\
\hline $4-6$ & 4934 & 99 & 4681 & 62 & $1.6(1.0$ to 2.5$)$ \\
\hline $0-3$ & 22055 & 67 & 22874 & 45 & $1.5(1.2$ to 1.9$)$ \\
\hline
\end{tabular}

Columns may not add up to total No because of missing data

${ }^{\star}$ Cumulative injury rate/10 000 children ages 3-24 months.

†Estimate of common relative risk (Mantel-Haenszel).

$\ddagger$ Maternal education was available on the birth certificate beginning in 1992. From 1992-95 11\% of the data were missing.
Table 3 Final multiple binomial regression model: independent predictors of increased risk of injury hospitalization or death due to injury between 3 and 24 months of age

\begin{tabular}{lll}
\hline Predictor of injury & Adjusted RR & $95 \%$ CI \\
\hline $\begin{array}{l}\text { Hospitalized }<90 \text { days old } \\
\quad \text { Yes }\end{array}$ & 1.5 & 1.2 to 1.8 \\
$\quad$ No & Referent & \\
Maternal age & & \\
$11-19$ & 5.4 & 3.0 to 9.5 \\
$20-24$ & 3.7 & 2.2 to 6.4 \\
$25-34$ & 2.0 & 1.2 to 3.4 \\
$35+$ & Referent & \\
Parity & & \\
$2+$ & 2.1 & 1.2 to 2.1 \\
1 & 1.6 & 1.6 to 2.8 \\
0 & Referent & \\
\hline
\end{tabular}

age than infants not hospitalized early (adjusted RR 1.5 ; 95\% CI 1.2 to 1.8 ). Infants hospitalized early who also had young mothers were at highest risk of serious injury, at least eight times the risk of the lowest risk group of infants, those without an early hospitalization with a nulliparous mother age 35 or older. The mother's occupation, level of education, insurance status, and race were not important confounders or independent predictors of a child's injury risk in this dataset and were therefore not included in the final regression model.

We also examined the association between hospitalization before 90 days of age and subsequent risk of injury by intent of injury (table 4). Full term infants who were hospitalized before 90 days of age for any reason were more than three times as likely to have a subsequent hospitalization for intentional injury than infants not hospitalized early (RR 3.3; 95\% CI 1.1 to 10.1 ). The rates of hospitalization for intentional injury were 4.4/10 000 in the early hospitalized group and 1.3/10 000 in the infants not hospitalized early.

Finally, we examined the association between the diagnosis of the first hospitalization at less than 90 days of age on the risk of subsequent injury, again compared to those infants who did not have an early hospitalization (table 5). Infants hospitalized in the first three months for bronchiolitis or pneumonia had more than a twofold increased risk of subsequent serious injury when compared with those not hospitalized early (RR 2.3; 95\% CI 1.7 to 3.1 ). The risk of subsequent injury for infants hospitalized for non-respiratory infectious diseases was nearly twice that of infants without an early hospitalization (RR 1.8; 95\% CI 1.2 to 2.5). Few infants were hospitalized before 90 days of age with an injury diagnosis and were therefore included in the group "other". When infants hospitalized with jaundice were excluded from the early hospitalized group, the relative risk of subsequent serious injury with early hospitalization for any reason other than jaundice increased to 1.9 (95\% CI 1.5 to 2.3). There were not enough intentional injuries to determine if the reason for the first admission influenced the risk associated with intentional injury in this dataset. Of the 13 intentional injuries in the early hospitalized group, five were initially hospitalized for an infection, four had bronchiolitis, two had 
Table 4 Risk of injury hospitalization or death by mechanism of injury among healthy full term infants hospitalized in the first three months of life relative to infants not hospitalized early

\begin{tabular}{llll}
\hline & $\begin{array}{l}\text { Early hospitalization } \\
(29466 \text { children })\end{array}$ & $\begin{array}{l}\text { No early hospitalization } \\
(29750 \text { children })\end{array}$ & $R R$ (95\% CI) \\
\hline Outcome & $13 / 10$ 000) & $4(1.3)$ & $3.3(1.1$ to 10.1$)$ \\
\hline Intentional injury & $177(59.7)$ & $111(37.3)$ & $1.6(1.3$ to 2.0$)$ \\
Unintentional injury & $35(11.8)$ & $26(8.7)$ & $1.4(0.8$ to 2.3$)$ \\
\hline Undetermined/no E code & &
\end{tabular}

Table 5 Effect of reason for first admission on risk of subsequent serious injury between 3 and 24 months of age

\begin{tabular}{lllll}
\hline Reason for hospitalization & $\begin{array}{l}\text { No with } \\
\text { diagnosis }\end{array}$ & $\begin{array}{l}\text { \% Of early } \\
\text { admissions }\end{array}$ & No of injuries & RR (95\% CI) \\
\hline Any diagnosis & 29466 & 100 & 225 & $1.6(1.3$ to 2.0) \\
Pneumonia/bronchiolitis & 5792 & 19.5 & 63 & $2.3(1.7$ to 3.1) \\
Other infectious disease & 4452 & 15.0 & 37 & $1.8(1.2$ to 2.5) \\
Perinatal conditions & 5625 & 19.0 & 38 & $1.4(1.0$ to 2.0) \\
Jaundice & 6657 & 22.4 & 25 & $0.8(0.5$ to 1.2$)$ \\
Other & 6940 & 23.6 & 62 & 1.8 (1.4 to 2.5) \\
No initial hospitalization & 29750 & & 141 & 1.0 (referent) \\
\hline
\end{tabular}

$\chi^{2}$ test for differences in injury rates between diagnosis groups in the early hospitalized cohort $\mathrm{p}<0.001$.

Table 6 Age of injury, length of stay and total charges by exposure status

\begin{tabular}{|c|c|c|c|}
\hline & $\begin{array}{l}\text { Prior early } \\
\text { hospitalization } \\
\text { Median }\end{array}$ & $\begin{array}{l}\text { No prior early } \\
\text { hospitalization } \\
\text { Median }\end{array}$ & p Value \\
\hline Age of injury & 14.7 months & 14.8 months & 0.20 \\
\hline Length of stay & 2.0 days & 2.0 days & 0.22 \\
\hline Total charges & $\$ 2314$ & $\$ 2146$ & 0.82 \\
\hline
\end{tabular}

$\star$ Wilcoxon rank sum test.

gastroenteritis, one was burned, and one had jaundice.

The median age at admission for the injury hospitalization, the length of the injury hospitalization, and the total charges for the injury hospitalization were similar between the group of infants hospitalized before 90 days of age and infants not hospitalized early (table 6).

\section{Discussion}

Although hospitalization or death due to injury in early childhood is relatively rare, nearly $20 \%$ of children visit an emergency department or clinic for injuries in this age group. ${ }^{11}$ This makes early childhood injuries an important and costly public health problem. In our study, full term infants hospitalized before 90 days of age had a $50 \%$ increased risk of future serious injury in early childhood. Infants hospitalized early were also three times more likely to be hospitalized for abuse before 24 months of age. This risk appears to be independent of known, measurable risk factors for early childhood injury mortality such as male gender, young maternal age, low socioeconomic markers, and high parity. Although previous studies have demonstrated that abused children are more likely to be hospitalized for medical conditions unrelated to abuse, ${ }^{89}$ no population based study has shown an association between early hospitalization and future increased risk of unintentional injury as well.

The reason for the first admission affected the risk of future injury. Most importantly, infants hospitalized for jaundice were at no increased risk of subsequent serious injury, whereas those hospitalized with a respiratory
Key points

- Full term infants hospitalized before 90 days of age for any reason other than jaundice are at increased risk for serious injury in early childhood.

- Health professionals should incorporate injury prevention anticipatory guidance into their discharge instructions for these infants.

- Hospitalized infants are easily identifiable for injury prevention interventions, and public health interventions could be specifically developed for this group of infants.

condition were more than twice as likely to have a serious injury before 24 months of age. If we excluded infants hospitalized early for jaundice, the risk of future serious injury associated with early hospitalization for any other reason increased to nearly twofold that of infants not hospitalized early.

Early hospitalization was unlikely to be a direct cause of increased risk of injury. Instead it may be a surrogate marker for family characteristics including parenting skills, household income, adequacy of social support, and other factors that place children at higher risk for both hospitalization in early infancy for certain conditions and for subsequent serious injury. Alternatively, underlying chronic illness that predisposes to both early hospitalization and to subsequent injury may account for the increased risk.

Young maternal age and high parity have been shown to be markers for increased risk of death due to injury in early childhood. ${ }^{1212} \mathrm{We}$ have demonstrated that these are also important risk factors for injury hospitalization in early childhood. Infants born to mothers younger than age 20 were at high risk of injury, independent of early hospitalization. Injury prevention efforts, such as home visits by trained staff, should continue to be focused on young mothers during their pregnancies and after delivery.

The limitations of studies that rely on secondary datasets, such as birth and death certificate data and hospital discharge records, have been well described. ${ }^{12}$ Although we were able to demonstrate a significant increase in risk of hospitalization for documented intentional injury in infants hospitalized for any reason before 90 days of age, the rate of intentional injury in both groups was low. Furthermore, the number of injury hospitalizations where there was no determined mechanism was more than three times that of documented intentional injuries, and intentional injury in this age group is often underdiagnosed or underreported. Therefore, the increased risk of hospitalization for intentional injury after an early hospitalization in this cohort of infants should be interpreted with caution.

Several potential confounders such as access to care and provider characteristics were not in 
this dataset. Furthermore, determining socioeconomic status may be subject to considerable measurement error. Infants of higher socioeconomic status or with better access to care may be less likely to be hospitalized in the first three months of life for common childhood illnesses. These infants may or may not be less likely to be hospitalized for subsequent injury. However, the injury hospitalizations for the infants hospitalized early did not differ substantially from that of infants not hospitalized early, at least not in terms of length of stay, age at admission, and hospital charges accrued. We examined only the first four hospitalizations for infants between 3 and 24 months of age, thereby missing hospitalizations that may have occurred subsequently. This likely resulted in a slight underestimate of the true risk of injury in the first 24 months of life in both groups. Finally, the data were limited to hospitalizations that occurred in Washington State.

\section{Implications for prevention}

Infants hospitalized before 90 days of age are an easily identified and potentially targetable group of infants for injury prevention interventions. Home visits by nurses has been shown to be effective in reducing injury hospitalizations in other high risk groups of infants, and may be an effective intervention in infants discharged from the hospital in early infancy, particularly if lack of community resources prohibits intensive home visit programs for all infants with high risk environments. ${ }^{5}$ In addition, interventions such as home nurse visitation may have many other benefits including reductions in emergency department visits for injuries, reductions in crime, and time on welfare for socially disadvantaged families. ${ }^{13} 14$

Providers and public health professionals who care for infants who are hospitalized early, particularly infants of young mothers, should be aware of the increased risk for serious injury in this group of infants. Providers may wish to incorporate injury prevention counseling in their discharge instructions or at follow up outpatient visits. Future studies may determine the mechanism responsible for this increased risk of both intentional and unintentional injury in infants hospitalized early. In addition, public health interventions could be specifically developed to reduce injuries in this "captive" group of infants.

1 Scholer SJ, Mitchel EF Jr, Ray WA. Predictors of injury mortality in early childhood. Pediatrics $1997 ; 100(3 \mathrm{pt}$ 1):342-7.

2 Cummings $\mathrm{P}$, Theis $\mathrm{MK}$, Mueller BA, et al. Infant injury death in Washington State, 1981 through 1990 [see comments]. Arch Pediatr Adolesc Med 1994;148:1021-6.

3 Benedict MI, White RB, Cornely DA. Maternal perinatal risk factors and child abuse. Child Abuse Negl 1985;9:21724.

4 Brown J, Cohen P, Johnson JG, et al. A longitudinal analysis of risk factors for child maltreatment: findings of a 17-year prospective study of officially recorded and self-reported child abuse and neglect. Child Abuse Negl 1998;22:106578 .

5 Kitzman H, Olds DL, Henderson CR Jr, et al. Effect of prenatal and infancy home visitation by nurses on pregnancy outcomes, childhood injuries, and repeated childbearing. A randomized controlled trial [see comments]. FAMA 1997; 278: $644-52$

6 Olds DL, Henderson CR Jr, Tatelbaum R, et al. Improving the delivery of prenatal care and outcomes of pregnancy: a randomized trial of nurse home visitation [published erratum appears in Pediatrics 1986;78:138]. Pediatrics 1986;77: $16-28$.

7 Scholer SJ, Hickson GB, Ray WA. Sociodemographic factors identify US infants at high risk of injury mortality. Pediatrics 1999;103(6 pt 1):1183-8.

8 DiScala C, Sege R, Li G, et al. Child abuse and unintentional injuries: a 10-year retrospective [see comments]. Arch Pediatr Adolesc Med 2000;154:16-22.

9 Leventhal JM, Pew MC, Berg AT, et al. Use of health services by children who were identified during the postpartum period as being at high risk of child abuse or neglect. Pediperiod as being at high
atrics 1996;97:331-5.

10 Sherrod KB, O'Connor S, Vietze PM, et al. Child health and maltreatment. Child Dev 1984;55:1174-83.

11 Danseco ER, Miller TR, Spicer RS. Incidence and costs of 1987-1994 childhood injuries: demographic breakdowns. Pediatrics 2000;105(2): 27

12 Brenner RA, Overpeck MD, Trumble AC, et al. Deaths attributable to injuries in infants, United States, 19831991. Pediatrics 1999;103(5 pt 1):968-74.

13 Olds DL, Eckenrode J, Henderson CR Jr, et al. Long-term effects of home visitation on maternal life course and child abuse and neglect. Fifteen-year follow-up of a randomized trial [comment]. fAMA 1997;278:637-43.

14 Olds D, Henderson CR Jr, Cole R, et al. Long-term effects of nurse home visitation on children's criminal and antisocial behavior: 15-year follow-up of a randomized controlled trial [see comments]. $\mathscr{f} A M A$ 1998;280:1238-44. 